\title{
YOUNG INVESTIGATORS: The cardiovascular profile of the low renin phenotype in a population of African descent
}

\author{
LEBO GAFANE-MATEMANE \\ Hypertension in Africa Research Team \\ North-West University, South Africa
}

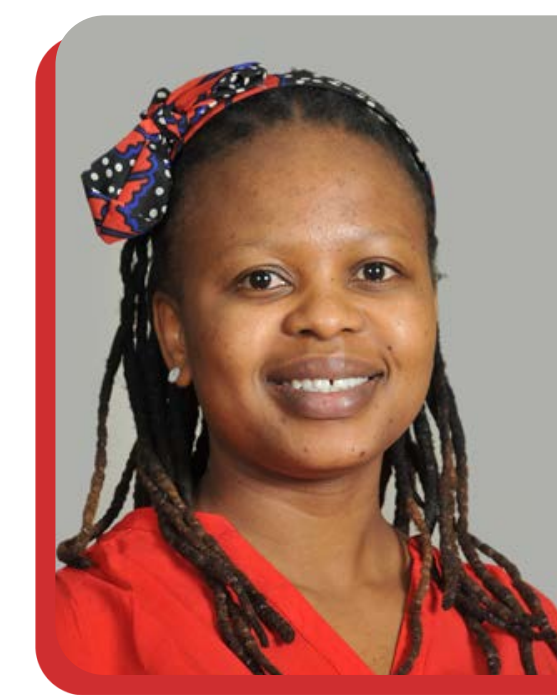

DOI: $10.30824 / 2006-16$

Populations of African ancestry (referred to as "black" hereafter) tend to present with a suppressed reninangiotensin-aldosterone system (RAAS) and severe forms of hypertension-mediated organ damage ${ }^{1}$. Relationships between components of the RAAS and cardiovascular disease are well established in other populations, however such information in Africa is scant. An attempt was made from the Sympathetic activity and Ambulatory Blood Pressure in Africans (SABPA) study to examine the cardiovascular profile of black South African school teachers. The study explored associations of renin and aldosterone with cardiovascular responses to stress and potential cardiac injury. The study population was aged 20-63 years and included both normotensives and hypertensives.

The main measurements included: (1) 24-hour systolic blood pressure (SBP), diastolic blood pressure (DBP) and heart rate (HR), from which percentage dipping was calculated, (2) a cold pressor test which was performed to measure cardiovascular and renin reactivity, (3) blood samples which were analysed for active renin, aldosterone, $\mathrm{N}$-terminal prohormone B-type natriuretic peptide (NTproBNP) as a measure of volume expansion, cardiac troponin $\mathrm{T}(\mathrm{cTnT})$ as measure of cardiomyocyte injury and copeptin as a surrogate measure of vasopressin. Basic characteristics of the population are described in Table 1.

\section{Main findings in the form of manuscripts}

\section{Plasma renin and cardiovascular responses to the cold pressor test differ in black and white populations}

Despite lower levels of renin (compared to white teachers) at rest and during stress, renin associated positively with vascular resistance only in black teachers when the cardiovascular system was challenged with the cold pressor test.

\section{Aldosterone and renin in relation to} surrogate measures of sympathetic activity

- Aldosterone and its ratio to renin were associated with less dipping of night-time HR, while renin showed a positive association with 24-hour HR only in the black population [3].

- There was also a borderline significant ( $P=0.051$ ) positive association between aldosterone and norepinephrine in the black group.

- It is therefore probable that aldosterone's direct effects and its relation to the sympathetic nervous system may impact cardiovascular function adversely in low renin states.

Of note: aldosterone levels were higher in the white group, however, the black group had a higher aldosterone-to-renin ratio (ARR).

3. Relation of the renin-angiotensinaldosterone system with potential cardiac injury and remodelling

- A marker of cardiomyocyte injury (CTnT) was positively associated with renin and a surrogate measure of vasopressin (copeptin).

- NTproBNP, an indicator of cardiac stress due to volume expansion was positively associated with copeptin 4 .

- Possible factors driving the association between cTnT and renin could be a pre-existing pressure and volume overload, as well as constant sympathetic activation due to emotional distress, which is well-established in the SABPA cohort 5 . 


\begin{tabular}{|c|c|c|c|}
\hline Manuscript 1 & Black $(\mathrm{N}=153)$ & White $(\mathrm{N}=188)$ & $P$ \\
\hline Age (years) & $43.1 \pm 7.71$ & $43.9 \pm 10.6$ & 0.38 \\
\hline Women, n (\%) & $73(47.7)$ & $99(52.7)$ & 0.36 \\
\hline Office SBP (mmHg) & $140 \pm 17.6$ & $130 \pm 13.3$ & $<0.001$ \\
\hline Office DBP (mmHg) & $81.1 \pm 9.83$ & $76.3 \pm 7.82$ & $<0.001$ \\
\hline Renin $(p g / m l)$ & $4.19 \pm 2.62$ & $6.32 \pm 3.07$ & $<0.001$ \\
\hline Manuscript 2 & Black ( $N=127)$ & White ( $N=179)$ & $P$ \\
\hline 24-hour SBP (mmHg) & $132 \pm 16.8$ & $125 \pm 12.9$ & $<0.001$ \\
\hline 24-hour DBP (mmHg) & $83.2 \pm 11.2$ & $77.0 \pm 8.20$ & $<0.001$ \\
\hline 24-hour HR (bpm) & $79.3 \pm 9.70$ & $73.7 \pm 10.2$ & $<0.001$ \\
\hline \% dipping SBP $(\mathrm{mmHg})$ & $10.1 \pm 6.34$ & $12.2 \pm 6.33$ & 0.003 \\
\hline \% dipping DBP (mmHg) & $15.9 \pm 11.9$ & $18.2 \pm 7.90$ & 0.042 \\
\hline \% dipping HR (bpm) & $14.6 \pm 8.78$ & $17.2 \pm 9.25$ & 0.015 \\
\hline Renin (pg/ml) & $3.47(0.95 ; 9.33)$ & $5.75(2.24 ; 12.3)$ & $<0.001$ \\
\hline Aldosterone (pg/ml) & $43.7(10.5 ; 170)$ & $55.0(21.8 ; 219)$ & 0.015 \\
\hline Aldosterone-to-renin ratio & $12.5(2.95 ; 67.6)$ & $9.55(2.88 ; 33.1)$ & 0.007 \\
\hline Manuscript 3 & Black (N=162) & White $(\mathrm{N}=206)$ & $P$ \\
\hline 24-hour SBP (mmHg) & $131 \pm 13.7$ & $124 \pm 10.4$ & $<0.001$ \\
\hline 24-hour DBP (mmHg) & $83 \pm 10.7$ & $77 \pm 8.04$ & $<0.001$ \\
\hline 24-hour HR (bpm) & $79 \pm 10.7$ & $72.0 \pm 9.55$ & $<0.001$ \\
\hline Hypertensive status, n (\%) & $87(62.6)$ & $66(39.8)$ & $<0.001$ \\
\hline Cardiac troponin T (ng/L) & $6.26(2.58 ; 8.64)$ & $5.66(2.67 ; 10.6)$ & 0.015 \\
\hline NTproBNP (pg/ml) & $30.4(7.20 ; 116)$ & $32.8(9.46 ; 114)$ & 0.47 \\
\hline Copeptin (pmol/L) & $4.95(2.02 ; 12.8)$ & $4.71(2.05 ; 12.4)$ & 0.38 \\
\hline Renin $(p g / m l)$ & $3.40(0.95 ; 9.24)$ & $5.83(1.88 ; 14.6)$ & $<0.001$ \\
\hline Aldosterone (pg/ml) & $44.9(10.2 ; 197)$ & $52.3(22.0 ; 151)$ & 0.078 \\
\hline Aldosterone-to-renin ratio & $13.2(2.9 ; 61.1)$ & $8.96(2.68 ; 27.2)$ & $<0.001$ \\
\hline
\end{tabular}

Overall, the SABPA study showed that even in low renin states, sympathetic dominance due to stress may contribute to the adverse associations between some components of the RAAS and cardiovascular structure and function. Raised BP and volume retention may increase the vulnerability to cardiac injury as a result of RAAS dysregulation and interactions with other pathways involved in volume regulation. In addition to physiological factors, socioeconomic and psychosocial factors such as chronic stress may contribute to the unfavourable cardiovascular profile in black populations with a suppressed RAAS.

There is a need to further our understanding of the complex mechanisms involved in the pathophysiology of hypertension in black populations, particularly the low renin phenotype. Impactful knowledge can be generated by performing studies that quantify salt sensitivity and profile the entire RAAS, including angiotensin converting enzyme 2 (ACE-2)Angiotensin-(1-7)-Mas receptor axis and angiotensin II type 2 receptor pathway. Evidence regarding the effect of tissue RAS on circulating RAAS and $B P$ regulation may shed light on the mechanisms that lead to raised BP when the circulating RAAS is suppressed. Such data have the potential to facilitate the development of efficient ethnicityspecific and/or personalised treatment approaches for hypertension.

Lebo Gafane-Matemane - lebo.gafane@nwu.ac.za

\section{REFERENCES}

1. Jane Lindhorst NA, Juliet Blignaut and Brian Rayner. Differences in hypertension between blacks and whites: an overview. Cardiovasc J Afr. 2007; 18:241-247.

2. Gafane LF, Schutte R, Van Rooyen JM, et al. Plasma renin and cardiovascular responses to the cold pressor test differ in black and white populations: The SABPA study. J Hum Hypertens. 2016; 30:346-351. doi: 10.1038/ jhh.2015.88

3. Gafane-Matemane LF, Van Rooyen JM, Schutte R, et al. Aldosterone and renin in relation to surrogate measures of sympathetic activity. Cardiovasc J Afr. 2019; 30:34-40.

4. Gafane-Matemane LF, Mokae NL, Breet Y, et al. Relation of the renin-angiotensin-aldosterone system with potential cardiac injury and remodelling: the SABPA study. Blood Press. 2020; 29:31-38. doi: 10.1080/08037051.2019.1645587

5. Malan L, Hamer M, Frasure-Smith N, et al. Cohort profile: Sympathetic activity and Ambulatory Blood Pressure in Africans (SABPA) prospective cohort study. Int J Epidemiol. 2015; 44:1814-1822. doi: 10.1093/ije/dyu199 